\title{
Internal tandem duplication and tyrosine kinase domain mutations in FLT3 alter the response to daunorubicin in $\mathbf{B a} / \mathbf{F} 3$ cells
}

\author{
SHINICHIRO TAKAHASHI and KUMI SHIRAHAMA \\ Division of Hematology, Kitasato University School of Allied Health Sciences, Kanagawa 252-0373, Japan
}

Received September 1, 2015; Accepted October 26, 2015

DOI: $10.3892 /$ br.2015.541

\begin{abstract}
Internal tandem duplication (ITD) and activating point mutations, mainly at aspartic acid 835 in the tyrosine kinase domain (TKD), are frequently identified in the Fms-related tyrosine kinase 3 (FLT3) receptor gene in acute myeloid leukemia. The ITD in FLT3 (FLT3-ITD) confers resistance to several chemotherapeutic drugs; however, the relative effects of FLT3-ITD and FLT3-TKD mutations on the efficacy of these drugs have not been reported. In the present study, ITD or TKD mutant forms of FLT3 in Ba/F3 cells were expressed, as in the absence of interleukin-3 (IL-3) the growth of these cells is completely dependent on FLT3 oncogenic signals. As a result, the $50 \%$ effective dose for daunorubicin was significantly higher in both Ba/F3-FLT3-ITD clones, and also in one of the two Ba/F3-FLT3-TKD clones when cells were cultured without IL-3. This phenomenon was not observed for cytarabine in either Ba/F3-FLT3-ITD or Ba/F3-FLT3-TKD cells. Collectively, these results indicate that ITD and TKD mutations in FLT3 may confer daunorubicin resistance in $\mathrm{Ba} / \mathrm{F} 3$ cells.
\end{abstract}

\section{Introduction}

Fms-like tyrosine kinase 3 (FLT3) is a member of the class III receptor tyrosine kinase receptor family (1) and is the most frequently mutated gene (20-30\%) in acute myelogenous leukemia (AML) (2-4). Activating mutations, as well as overexpression of FLT3, are prevalent in AML, with a role in leukemogenesis $(3,5-8)$. In adult AML, $24 \%$ of such mutations are internal tandem duplications (ITD) in the juxtamembrane domain $(2,3)$, which result in ligand-independent dimerization and tyrosine phosphorylation of the receptor (9). In addition, activating point mutations in the FLT3 tyrosine kinase domain

Correspondence to: Professor Shinichiro Takahashi, Division of Hematology, Kitasato University School of Allied Health Sciences, 1-15-1 Kitasato, Minami, Sagamihara, Kanagawa 252-0373, Japan E-mail: shin@kitasato-u.ac.jp

Key words: FLT3-internal tandem duplication, FLT3-tyrosine kinase domain, daunorubicin, drug resistance, Ba/F3 cells
(TKD; FLT3-TKD), mainly at aspartic acid 835, are identified in $\sim 7 \%$ of AML patients (10).

ITD in FLT3 (FLT3-ITD) is associated with a higher leukocyte count, increased relapse risk, decreased disease-free survival (DFS) and decreased overall survival (OS) (11). Furthermore, multivariate analyses have shown that FLT3-ITD is the most significant factor for predicting an adverse outcome in AML (11-14). By contrast, FLT3-TKD mutations have a smaller effect compared with FLT3-ITD, but tend to worsen the DFS and OS (10), with the differences being statistically significant for OS in patients aged $\leq 60$ years (15).

FLT3-ITD alters chemotherapy responses in vitro and in vivo and confers resistance to doxorubicin, which depends on p53 (16). Additionally, DNA repair contributes to the FLT3-ITD drug-resistant phenotype of primary AML (17). Several studies demonstrate drug resistance conferred by FLT3-ITD $(16,18,19)$; however, thus far, no studies have demonstrated the effect of mutations in the FLT3 TKD on anticancer drug resistance.

The present study examined the effects of FLT3-ITD and FLT3-TKD on cytotoxic drugs by employing an IL-3 dependent cell line, $\mathrm{Ba} / \mathrm{F} 3$. In this cell line, interleukin-3 (IL-3)-independent cell growth occurs in response to stably transduced oncogenic signaling, such as via FLT3-ITD and -TKD (20). This system was used to evaluate the effect of FLT3-ITD and -TKD oncogenic signals on the cytotoxicity of daunorubicin (DNR) and cytarabine (Ara-C). As a result, FLT3-ITD and -TKD signals were observed to alter the response to DNR.

\section{Materials and methods}

Generation of Ba/F3 cells expressing FLT3-ITD and FLT3-TKD. Our previous studies established the Ba/F3 FLT3-WT and-ITD cells $(6,21)$. To generate stably expressing Ba/F3-FLT3-TKD cells, pcDNA FLT3-TKD (D835Y) was generated using pcDNAFLT3-WT (6) and the Quick change site directed mutagenesis kit XL (Stratagene, La Jolla, CA, USA) with the following primers: Sense, 5'-CTTTGGATT GGCTCGATATATCATGAGTGATTC-3' and anti-sense, 5'-GAATCACTCATGATATATCGAGCCAATCCAAAG-3'.

The construct was confirmed by sequence analysis and transfected into Ba/F3 cells using a CLB-Transfection device (Lonza, Basel, Switzerland). Stably transfected Ba/F3 clones 
were isolated by limiting dilution and selection with $400 \mu \mathrm{g} / \mathrm{ml}$ neomycin in RPMI (Gibco BRL, Thermo Fisher Scientific, Rockville, MD, USA) containing $10 \%$ heat-inactivated fetal bovine serum, $100 \mathrm{ng} / \mathrm{ml}$ recombinant mouse IL-3 (R\&D Systems, Minneapolis, MN, USA) and $50 \mu \mathrm{mol} / 1$ 2-mercaptoethanol. Cells were cultured at $37^{\circ} \mathrm{C}$ in a humidified $5 \% \mathrm{CO}_{2}$ atmosphere.

mRNA analysis. cDNA was prepared from cells using reverse transcriptase (Transcriptor First Strand cDNA Synthesis kit; Roche, Indianapolis, IN, USA). Reverse transcription-quantitative polymerase chain reaction (RT-qPCR) was performed using the Quantitect SYBR-Green PCR reagent (Qiagen, Miami, FL, USA) according to the manufacturer's protocol and using an Opticon Mini Real-time PCR Instrument (Bio-Rad, Hercules, CA, USA), as described previously (22). The primer sequences were: FLT3 forward, 5'-TCAAGTGCTGTGCATACAATT CCC-3' and reverse, 5'-CACCTGTACCATCTGTAGCTG GCT-3'; and GAPDH forward, 5'-GAAGGTGAAGGTCGG AGT-3' and reverse, 5'-GAAGATGGTGATGGGATTTC-3'. The thermal cycling conditions for FLT3 and GAPDH were incubation at $95^{\circ} \mathrm{C}$ for $15 \mathrm{~min}$, followed by 35 cycles of $95^{\circ} \mathrm{C}$ for $30 \mathrm{sec}, 55^{\circ} \mathrm{C}$ for $30 \mathrm{sec}$ and $72^{\circ} \mathrm{C}$ for $45 \mathrm{sec}$. The copy number of each sample was calculated as previously described (21).

Assessment of viable cells. The proportion of viable cells was determined using a dye reduction assay involving a tetrazolium salt, 2-(2-methoxy-4-nitrophenyl)-3-(4-nitrophenyl)-5-(2,4-disulfophenyl)-2H-tetrazolium, monosodium salt (WST-8; Dojindo, Tokyo, Japan), which is a modification of the 3-(4,5-dimethylthiazol-2-yl)-2,5-diphenyltetrazolim assay. The effective dose $(E D)_{50}$ values were calculated from the data obtained from the cell growth assays. Exponentially growing cells were seeded at $1 \times 10^{4}$ cells per well in flat-bottomed 96-well plates, with or without IL-3 in the medium. Seven different doses were selected for DNR (Sigma, St. Louis, MO, USA) (1.5, 3.1, 6.2 , 12.5, 25, 50 and $100 \mathrm{nM}$ ) and Ara-C (Sigma) (0.15, 0.31, $0.62,1.25 .2 .5,5$ and $10 \mu \mathrm{M})$. No DNR or Ara-C was added to the controls cells. Assays were performed 2 days after the addition of the drugs. For IL-3(-) cells, exponentially growing cells were washed twice with phosphate-buffered saline and seeded without IL-3. Viable cells (\%) were calculated as the ratio of the absorbance $(490 \mathrm{~nm})$ of DNR or Ara-C-treated cells to the absorbance of untreated cells. At least three independent experiments were performed. The calculated ratios were analyzed and the $\mathrm{ED}_{50}$ values were obtained using tools at http://www.vector. co.jp/soft/win95/edu/se248471.html.

Statistical analysis. Data are expressed as the mean \pm standard error of the mean and $\mathrm{P}<0.05$ (denoted by one asterisk) was considered to indicate a statistically significant difference. Comparison of the means was performed using Student's t-test (http://www.physics.csbsju.edu/stats/t-test_bulk_form.html).

\section{Results}

Generation of Ba/F3-FLT3-TKD cells. Our previous study established the generation of Ba/F3-FLT3-ITD cells (21), and for the present study, to clarify whether FLT3-ITD or FLT3-TKD mediate any specific anticancer drug effects,

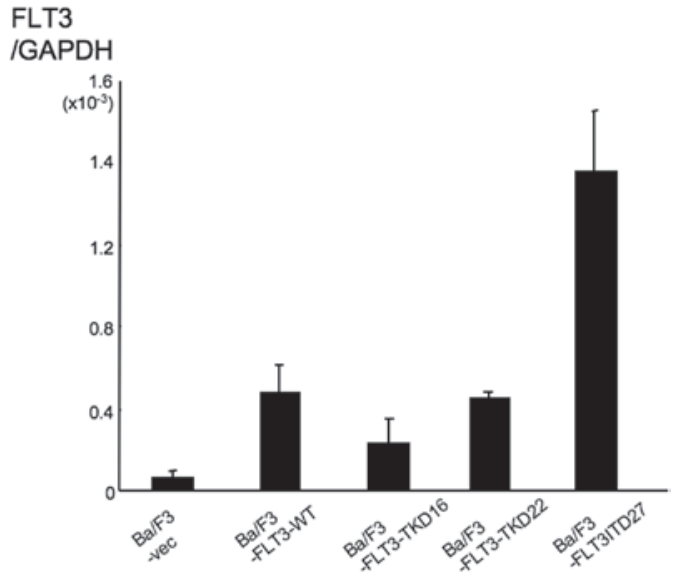

Figure 1. Expression of FLT3 transgene in $\mathrm{Ba} / \mathrm{F} 3$ cells. Reverse-transcription quantitative polymerase chain reaction (PCR) analyses of FLT3 using newly established Ba/F3-FLT3-TKD16 and Ba/F3-FLT3-TKD22 lines and the previously generated Ba/F3-vec, Ba/F3-FLT3-WT (6) and Ba/F3-FLT3-ITD27 (21) lines. FLT3 transcript levels were adjusted relative to the expression of GAPDH. The data presented were obtained from three independent PCR amplifications.

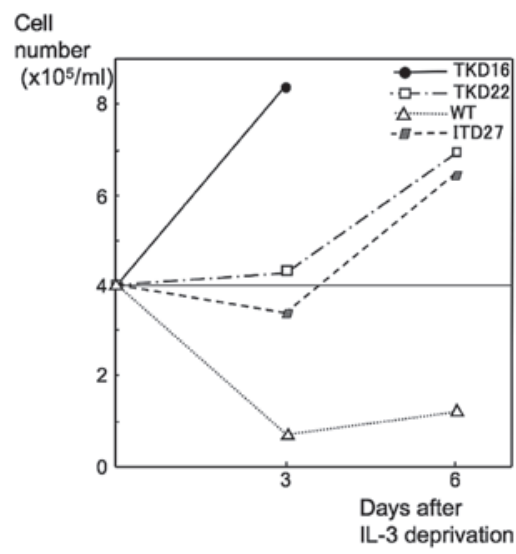

Figure 2. Effect of the FLT3-TKD transgene on the growth of $\mathrm{Ba} / \mathrm{F} 3$ cells To measure cell proliferation, Ba/F3-FLT3-TKD (TKD16, TKD22) cells together with $\mathrm{Ba} / \mathrm{F} 3-F L T 3-\mathrm{WT}$ and $\mathrm{Ba} / \mathrm{F} 3-F L T 3-\mathrm{ITD}$ (ITD27) cells were counted at days 3 and 6 following IL-3 deprivation, using a hemocytometer and the trypan blue exclusion method. The plotted values were obtained from an average of four counts, and the result is a representative of two independent experiments. IL-3, interleukin-3.

$\mathrm{Ba} / \mathrm{F} 3-F L T 3-\mathrm{TKD}$ cells were also generated. The pcDNA FLT3-TKD (D835Y) vector was electroporated into Ba/F3 cells and stably transfected lines were isolated by limiting dilution with medium containing neomycin. Among >20 lines obtained, two clones, Ba/F3-FLT3-TKD16 and Ba/F3-FLT3-TKD22, exhibited increased levels of FLT3 expression compared with the parental vector (pcDNA3.1) transfected Ba/F3-vec cells (Fig. 1). To confirm that the FLT3-TKD transgene was functionally active, Ba/F3-FLT3-TKD16 and Ba/F3-FLT3-TKD22 cells were deprived of IL-3. These two lines showed factor-independent growth, as previously reported $(21,23)$. Similar growth was observed for Ba/F3-FLT3-ITD (positive control), but not for $\mathrm{Ba} / \mathrm{F} 3-$ FLT3-WT (negative control) (Fig. 2).

$E D_{50}$ of DNR increases in IL-3-deprived Ba/F3-FLT3-ITD27, -ITD29 and -TKD22 cells. Cell viability and $\mathrm{ED}_{50}$ values were 
A DNR

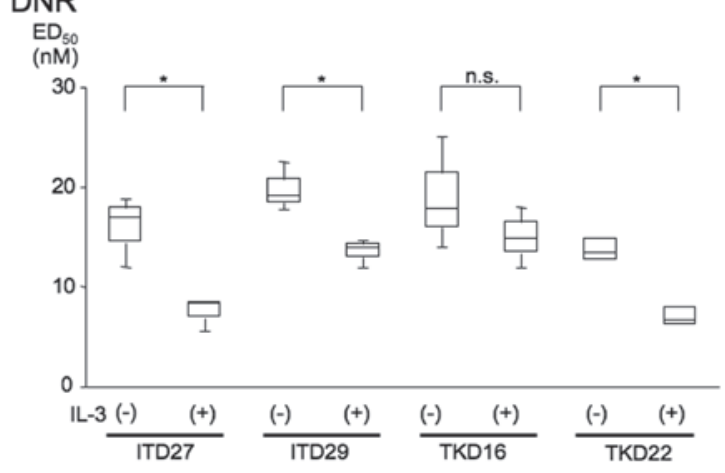

B

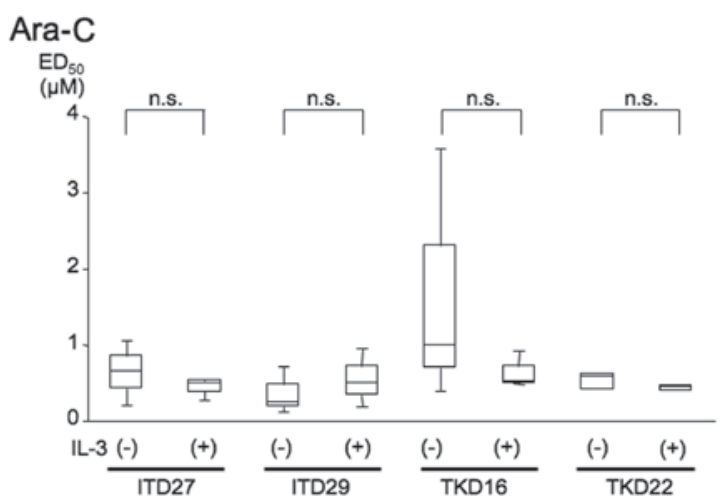

Figure 3. $\mathrm{ED}_{50}$ values for (A) DNR and (B) Ara-C. The $\mathrm{ED}_{50}$ values were calculated from the data obtained from the cell growth assays. WST-8 assays were performed on cells grown with or without IL-3 in the medium and 2 days after the addition of drugs: DNR $(1.5,3.1,6.2,12.5,25,50$ or $100 \mathrm{nM})$ and Ara-C $(0.15,0.31,0.62,1.25 .2 .5,5$ or $10 \mu \mathrm{M})$. Control cells were grown without the addition of DNR or Ara-C. Viable cells (\%) were determined by the ratio of the absorbance $(490 \mathrm{~nm})$ of DNR or Ara-C-treated cells relative to the absorbance of untreated cells. The ratio of viable treated cells to untreated cells was used to calculate $\mathrm{ED}_{50}$ values. At least three independent experiments were performed. These data are shown as boxplots representing the 25 and 75 percentiles, median and 5-95 range. Data are expressed as the mean \pm standard error of the mean and ${ }^{*} \mathrm{P}<0.05$ was considered to indicate a statistically significant difference. ED, effective dose; DNR, daunorubicin; IL-3, interleukin-3; n.s., not significant.

examined in the $\mathrm{Ba} / \mathrm{F} 3-F L T 3-\mathrm{ITD}$ and $\mathrm{Ba} / \mathrm{F} 3-F L T 3-\mathrm{TKD}$ cells. In Ba/F3-FLT3-ITD27, -ITD29 and -TKD22 cells, the $\mathrm{ED}_{50}$ value was significantly increased in the absence of IL-3, compared with the controls (Fig. 3A). Additionally, in $\mathrm{Ba} / \mathrm{F} 3-\mathrm{FLT3}-\mathrm{TKD} 16$ cells, the median value was higher [IL-3(-), $17.7 \mathrm{nM}$; IL-3(+), $14.8 \mathrm{nM}$ ], although this difference was not statistically significant (Fig. 3A). By contrast, there were no differences in the $\mathrm{ED}_{50}$ value for Ara-C between IL-3(-) and IL-3(+) cells (Fig. 3B). These results indicate that FLT3-ITD, as well as the mutation of the FLT3-TKD, may confer DNR resistance to $\mathrm{Ba} / \mathrm{F} 3$ cells.

\section{Discussion}

The present data suggest that in AML patients with FLT3-ITD or FLT3-TKD mutations, DNR is not efficacious and only causes toxicity. This is consistent with the findings of a clinical trial showing that while the majority of AML patients benefit from intensified anthracycline dosing regimens, high-dose DNR did not provide a significant survival benefit in patients who had the FLT3-ITD mutation (24).
Lee et al (25) also support this notion that FLT3-ITD causes resistance to doxorubicin; dual treatment of PML-RAR $\alpha$ FLT3-ITD transgenic mice with FLT3 inhibitor SU11657 and doxorubicin increased sensitivity. Pardee et al (16) recently reported that FLT3-ITD confers resistance to doxorubicin in a p53-dependent manner. In addition to the well-known role of p53 in apoptosis induction, it has also been shown to induce multiple prosurvival and DNA repair genes (26). Consistent with this, FLT3-ITD-expressing AML cell lines and primary patient samples have increased the levels of reactive oxygen species, double-strand DNA breaks and increased DNA repair capacity $(17,27)$.

The present findings are consistent with and support the above. However, using murine myeloid HF6 and human myeloid K562 cells, our previous study found that FLT3-ITD induced Ara-C resistance through repression of equilibrative nucleoside transporter 1 expression (19), which was not observed in the present study. This discrepancy may be due to the use of different cell lines. The present study employed $\mathrm{Ba} / \mathrm{F} 3$ cells, a murine $\mathrm{B}-1 y m p h o i d$ cell line, which is distinct from HF6 and K562 cells. The Ba/F3 cells were used due to their IL-3 deprivation characteristic, which can reveal the effect of FLT3 oncogenic signals. This enabled a specific DNR response to be identified.

The study also highlights the FLT3-TKD mutations, which may confer resistance to anthracycline. Although the data are from a cell line model and are somewhat preliminary, these results may indicate the benefit of therapy combining anthracycline and FLT3 inhibitors for patients carrying FLT3-ITD and FLT3-TKD mutations.

\section{Acknowledgements}

The authors would like to thank Mr. Tatsuya Osaka and Mr. Wataru Nomura for establishing the cell lines and Ms. Hiroko Nakano for her technical assistance. The study was supported in part by Grants-in-Aid for Scientific Research (grant no. 26460685) from the Ministry of Education, Science and Culture (Japan), and the Takeda Sceince Foundation, a foundation from Kitasato University School of Allied Health Sciences (Grant-in-Aid for Research Project, no. 2015-1003). Edanz Group Ltd. (www.edanzediting.com) provided editorial assistance.

\section{References}

1. Rosnet O, Matteï MG, Marchetto S and Birnbaum D: Isolation and chromosomal localization of a novel FMS-like tyrosine kinase gene. Genomics 9: 380-385, 1991.

2. Yokota S, Kiyoi H, Nakao M, Iwai T, Misawa S, Okuda T, Sonoda Y, Abe T, Kahsima K, Matsuo Y and Naoe T: Internal tandem duplication of the FLT3 gene is preferentially seen in acute myeloid leukemia and myelodysplastic syndrome among various hematological malignancies. A study on a large series of patients and cell lines. Leukemia 11: 1605-1609, 1997.

3. Gilliland DG and Griffin JD: The roles of FLT3 in hematopoiesis and leukemia. Blood 100: 1532-1542, 2002.

4. Takahashi S: Downstream molecular pathways of FLT3 in the pathogenesis of acute myeloid leukemia: Biology and therapeutic implications. J Hematol Oncol 4: 13, 2011.

5. Ozeki K, Kiyoi H, Hirose Y, Iwai M, Ninomiya M, Kodera Y, Miyawaki S, Kuriyama K, Shimazaki C, Akiyama $\mathrm{H}$, et al: Biologic and clinical significance of the FLT3 transcript level in acute myeloid leukemia. Blood 103: 1901-1908, 2004. 
6. Takahashi S, Harigae H, Ishii KK, Inomata M, Fujiwara T, Yokoyama H, Ishizawa K, Kameoka J, Licht JD, Sasaki T and Kaku M: Over-expression of Flt3 induces NF-kappaB pathway and increases the expression of IL-6. Leuk Res 29: 893-899, 2005.

7. Takahashi S, Harigae H, Kaku M, Sasaki T and Licht JD: Flt3 mutation activates p21 (WAF1/CIP1) gene expression through the action of STAT5. Biochem Biophys Res Commun 316 $85-92,2004$

8. Takahashi S, McConnell MJ, Harigae H, Kaku M, Sasaki T, Melnick AM and Licht JD: The Flt3 internal tandem duplication mutant inhibits the function of transcriptional repressors by blocking interactions with SMRT. Blood 103: 4650-4658, 2004.

9. Kiyoi H, Ohno R, Ueda R, Saito H and Naoe T: Mechanism of constitutive activation of FLT3 with internal tandem duplication in the juxtamembrane domain. Oncogene 21: 2555-2563, 2002.

10. Yamamoto Y, Kiyoi H, Nakano Y, Suzuki R, Kodera Y, Miyawaki S, Asou N, Kuriyama K, Yagasaki F, Shimazaki C, et al: Activating mutation of D835 within the activation loop of FLT3 in human hematologic malignancies. Blood 97: 2434-2439, 2001.

11. Kottaridis PD, Gale RE, Frew ME, Harrison G, Langabeer SE Belton AA, Walker H, Wheatley K, Bowen DT, Burnett AK, et al: The presence of a FLT3 internal tandem duplication in patients with acute myeloid leukemia (AML) adds important prognostic information to cytogenetic risk group and response to the first cycle of chemotherapy: Analysis of 854 patients from the United Kingdom medical research council AML 10 and 12 trials. Blood 98: 1752-1759, 2001.

12. Meshinchi S, Woods WG, Stirewalt DL, Sweetser DA, Buckley JD, Tjoa TK, Bernstein ID and Radich JP: Prevalence and prognostic significance of Flt3 internal tandem duplication in pediatric acute myeloid leukemia. Blood 97: 89-94, 2001

13. Kiyoi H, Naoe T, Nakano Y, Yokota S, Minami S, Miyawaki S, Asou N, Kuriyama K, Jinnai I, Shimazaki C, et al: Prognostic implication of FLT3 and N-RAS gene mutations in acute myeloid leukemia. Blood 93: 3074-3080, 1999

14. Frohling S, Schlenk RF, Breitruck J, Benner A, Kreitmeier S, Tobis K, Döhner H and Döhner K; AML Study Group Ulm. Acute myeloid leukemia: Prognostic significance of activating FLT3 mutations in younger adults (16 to 60 years) with acute myeloid leukemia and normal cytogenetics: A study of the AML Study Group Ulm. Blood 100: 4372-4380, 2002.

15. Thiede C, Steudel C, Mohr B, Schaich M, Schäkel U, Platzbecker U, Wermke M, Bornhäuser M, Ritter M, Neubauer A, et al: Analysis of FLT3-activating mutations in 979 patients with acute myelogenous leukemia: Association with FAB subtypes and identification of subgroups with poor prognosis. Blood 99: 4326-4335, 2002.
16. Pardee TS, Zuber J and Lowe SW: Flt3-ITD alters chemotherapy response in vitro and in vivo in a p53-dependent manner. Exp Hematol 39: 473.e4-485.e4, 2011.

17. Seedhouse CH, Hunter HM, Lloyd-Lewis B, Massip AM, Pallis M, Carter GI, Grundy M, Shang S and Russell NH: DNA repair contributes to the drug-resistant phenotype of primary acute myeloid leukaemia cells with FLT3 internal tandem duplications and is reversed by the FLT3 inhibitor PKC412. Leukemia 20: 2130-2136, 2006.

18. Zwaan CM, Meshinchi S, Radich JP, Veerman AJ, Huismans DR, Munske L, Podleschny M, Hählen K, Pieters R, Zimmermann M, et al: FLT3 internal tandem duplication in 234 children with acute myeloid leukemia: Prognostic significance and relation to cellular drug resistance. Blood 102: 2387-2394, 2003.

19. Jin G, Matsushita H, Asai S, Tsukamoto H, Ono R, Nosaka T, Yahata T, Takahashi S and Miyachi H: FLT3-ITD induces ara-C resistance in myeloid leukemic cells through the repression of the ENT1 expression. Biochem Biophys Res Commun 390: 1001-1006, 2009.

20. Spiekermann K, Bagrintseva K, Schwab R, Schmieja K and Hiddemann W: Overexpression and constitutive activation of FLT3 induces STAT5 activation in primary acute myeloid leukemia blast cells. Clin Cancer Res 9: 2140-2150, 2003.

21. Takahashi S, Harigae H, Kameoka J, Sasaki T and Kaku M AML1B transcriptional repressor function is impaired by the Flt3 internal tandem duplication. Br J Haematol 130: 428-436, 2005.

22. Iseki Y, Nakahara M, Kubo M, Obata F, Harigae $H$ and Takahashi S: Correlation of PU.1 and signal regulatory protein $\alpha 1$ expression in PU.1 transgenic K562 cells. Int J Mol Med 29: 319-323, 2012

23. Hayakawa F, Towatari M, Kiyoi H, Tanimoto M, Kitamura T, Saito $\mathrm{H}$ and Naoe T: Tandem-duplicated Flt3 constitutively activates STAT5 and MAP kinase and introduces autonomous cell growth in IL-3-dependent cell lines. Oncogene 19: 624-631, 2000.

24. Fernandez HF, Sun Z, Yao X, Litzow MR, Luger SM, Paietta EM, Racevskis J, Dewald GW, Ketterling RP, Bennett JM, et al: Anthracycline dose intensification in acute myeloid leukemia. N Engl J Med 361: 1249-1259, 2009.

25. Lee BD, Sevcikova S and Kogan SC: Dual treatment with FLT3 inhibitor SU11657 and doxorubicin increases survival of leukemic mice. Leuk Res 31: 1131-1134, 2007.

26. Janicke RU, Sohn D and Schulze-Osthoff K: The dark side of a tumor suppressor: Anti-apoptotic p53. Cell Death Differ 15: 959-976, 2008

27. Sallmyr A, Fan J, Datta K, Kim KT, Grosu D, Shapiro P, Small D and Rassool F: Internal tandem duplication of FLT3 (FLT3/ITD) induces increased ROS production, DNA damage and misrepair: Implications for poor prognosis in AML. Blood 111: 3173-3182, 2008. 